\title{
DENGUE DURING PREGNANCY: ASSOCIATION WITH LOW BIRTH WEIGHT AND PREMATURITY
}

Christiane Fernandes RIBEIRO(1), Vânia Glória Silami LOPES(2), Patricia Brasil(3), Licinio Esmeraldo da Silva(4), Pedro Henrique Fernandes Josephson RIBEIRO(5), Luca Cipriani UGENTI(5) \& Rita Maria Ribeiro NOGUEIRA(6)

\begin{abstract}
SUMMARY
The aim of this study was to evaluate the effects of dengue virus infection during pregnancy and its correlation with low birth weight, prematurity, and asphyxia. A non-concurrent cohort study reveals the association of dengue during pregnancy with prematurity and low birth weight, when birth occurred during the maternal-fetal viremia period ( $p=0.016$ and $p<0.0001$, respectively).
\end{abstract}

KEYWORD: Dengue; Pregnancy; Newborn; Prematurity; Low birth weight.

\section{INTRODUCTION}

Dengue, endemic in many tropical countries, is a viral disease transmitted by mosquitoes, classified into 4 serotypes $(1,2,3 \text { and } 4)^{1,2}$.

Various arboviruses are known to cause abortion, fetal death, premature labor, and teratogenic alterations in humans and animals ${ }^{3}$. The first report of pregnancy associated with dengue and described in the literature dates back to 1948 and reported the case of a pregnant woman infected in the beginning of gestation, which evolved without any intercurrences ${ }^{4}$. However, over the years, some authors have reported the effects of DENV infection in pregnant women with severe consequences for fetuses and newborns $5,6,7,8$.

The aim of this study was to evaluate the effects on the conceptus caused by the DENV infection during pregnancy, associated with low birth weight, prematurity, and perinatal asphyxia.

\section{METHOD AND MATERIALS}

This is a non-concurrent cohort study in pregnant women and their respective conceptus, during 2008 in Rio de Janeiro. This study used two databases, SINAN/DENGUE/GESTANTE (2008) and SINASC (2008/2009) of Rio de Janeiro, and was approved by the Research Ethics Committee of the School of Medicine - Antônio Pedro University Hospital, of the Fluminense Federal University (CMM/HUAP N ${ }^{\circ} 038$ ).

Pregnant women without dengue infection and pregnant women with clinical-epidemiological and laboratorial diagnosis of dengue, notified in Rio de Janeiro State during the year of 2008, were included in this study (SINAN/DENGUE/GESTANTE 2008)
Pregnant women with DENV infection were defined as those matching the following criteria: Clinical-epidemiological: Pregnant woman with acute febrile illness of maximum duration of seven days, accompanied by at least two of the following symptoms: cephalea, retro-orbital pain, myalgia, arthralgia, prostration, exanthema, associated with epidemiological history (resident of neighborhoods with confirmed epidemic or having visited locations with confirmed epidemic). Laboratorial: IgM testing with the MAC-ELISA method with a positive result or positive viral isolation or positive PCR for DENV.

Neonates were defined as premature if gestational age was inferior to 37 weeks, and low weight if birth weight was inferior to $2,500 \mathrm{~g}^{9,10}$. Neonates with asphyxia were defined as having an Apgar score at the fifth minute of life lower than $7^{11}$.

Damage to the conceptus was defined as the occurrence of premature labor, low birth weight, or perinatal asphyxia.

Considering five to six days of maternal viremia ${ }^{12}$, three to five days of incubation for the fetus, and five to six days of viremia in the fetus, maternal-fetal viremia period (MFVP) was defined as the period between the first day of disease in the pregnant woman (D1) and the date of labor equal or inferior to fifteen days.

The Reclink II software ${ }^{13}$ was used to associated data from the SINAN/DENGUE/GESTANTE 2008 and the SINASC 2008/2009, and to obtain the information related to the birth of neonates from mothers that presented the date of the first signs and symptoms during pregnancy.

The following variables were used to associate the SINAN/DENGUE/ GESTANTE 2008 and the SINASC 2008/2009, with distinct functions 


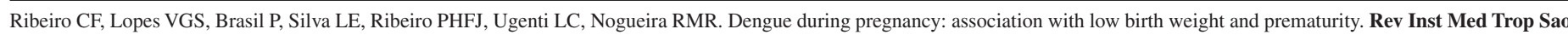
Paulo. 2016;58:8.

(Key field, Blocking), to decide the true pair and also for data analysis:

a) in SINASC: NUMERODN (Key field), ENDNASC, CODMUNNASC, BAINASC, LOCNASC, NOMEMAE, IDADEMAE, CODMUNIRES, BAIRES, ENDRES, COMPLRES, NUMRES, GESTAÇÃO, DTNASC, HORA, SEXO, APGAR1, APGAR5, PESO, GRAVIDEZ, PARTO;

b) in SINAN: NU_NOTIFIC (Key field), DT_NOTIFIC, ID_ MUNICIP, DT_SIN_PRI, NM_PACIENT, DT_NASC, NU_IDADE_N, CS_GESTANT, NM_MAE_PAC, ID_MN_RESI, NM_BAIRRO, NM_ LOGRADO, NU_NUMERO, NU_COMPLEM, NU_DDD_TEL, NU_ TELEFON, DT_SORO, RESULT_SORO,DT_VIRAL, RESUL_VI_N, SOROTIPO, IMUNO_N, DT_PCR, RESUL_PCR, CLASSIF_FIN, CRITERIO, EVOLUÇÃO, DT_OBITO and DT_ENCERRA.

The standardization routine utilized NOMEMAE (mother's name) from SINASC and NM_PACIENT (patient's name) from SINAN, which were split into first name and last name.

For the blocking, logistical blocks of records were created for further relating, implementing the SOUDEX (phonetic code) for this purpose, because it presents the lowest chance of error than using directly the first and last names.

The Reclink II software, a free software published by the Free Software Foundation (Borland International Inc., 1998a; Reisdorph, 1998). , obtained a calculation of scores that summarize the degree of global agreement between records of a same pair. It was considered to be true the pair with scores higher than $5.55^{13}$.

The last step consisted of checking the combined file that was saved in the Excel software. The fields used for the decision of the true pair were: CODMUNIRES (SINASC)/ ID_MN_RESI (SINAN); ENDRES, COMPLRES, NUMRES(SINASC)/ NM_LOGRADO, NU_NUMERO, NU_COMPLEM (SINAN); IDADEMAE(SINASC)/ DT_NASC(SINAN), in that order.

After associating the databases and checking them, it was obtained a combined file of 336 true pairs, that is, pregnant woman with dengue notified in SINAN/DENGUE, and with a declaration of live birth in SINASC. These 336 true pairs were divided into neonates born in the maternal-fetal viremia period, and neonates born after the maternal-fetal viremia period.
For statistical analysis, three groups were taken into consideration: 1) neonates from mothers with dengue born in the maternal-fetal viremia period, 2) neonates from mothers with dengue born outside the maternal-fetal viremia period, and 3) neonates born from a mother without DENV infection (SINASC 2008 database, excluding the 336 true pairs). Comparison between the three groups was performed by the chi-square test. A $P$-value $<0.05$ was set as significant in all the analyses.

\section{RESULTS}

In the study period (January 2008 to January 2009), from the 345,935 births occurred in Rio de Janeiro (SIM), 336 (0.97\%) were from pregnant women who had dengue (SINAN-RJ). Thirty-five births occurred within the MFVP and 281 occurred outside the MFVP. We compared the frequency of prematurity and low birth age between pregnant women of these two groups.

Premature births were more frequent amongst neonates born during the MFVP (6/41-14.6\%) than in those neonates born after the MFVP $(14 / 295-4.7 \%)$. This difference was statistically significant $(p=0.016)$. The frequency of prematurity in this last group was similar to those found in mothers without dengue $(17,079 / 345,599)$ (Table 1).

Perinatal asphyxia occurred in one $(2.4 \%)$ out of the 41 neonates born during the MFVP and in three (1.0\%) out of the 295 neonates born out of the MFVP $(p=0.268)$. Amongst newborns from mothers without dengue, asphyxia occurred in $8,600(2.4 \%)$ out of the 354,599 cases.

Low birth weight was observed in nine $(22.0 \%)$ of the 41 neonates born during the maternal-fetal DEN viremia period, and in $13(4.4 \%)$ of the 295 neonates of mothers with dengue that were born after the MFVP. This difference was highly significant $(p<0.0001)$. Amongst mothers without dengue, low birth weight was observed in 18,017 (5.1\%) out of the 345,599 RN (Table 2).

\section{DISCUSSION}

The epidemiological analysis resulting from correlating the database of pregnant women with dengue (SINAN) in 2008 (when an epidemic of DENV 2 occurred in the Rio de Janeiro State) with the declarations of live birth (SINASC) in 2008 and 2009, presents evidence of the impact of the DENV infection in birth weight and gestational age, with a statistically significant increase of premature and neonates with birth weight inferior to $2,500 \mathrm{~g}(p<0.05)$, when the delivery occurred in a

Table 1

Association between dengue during gestation and gestational age

\begin{tabular}{|c|c|c|c|}
\hline \multirow{2}{*}{ Mother/Neonate } & \multicolumn{2}{|c|}{ Neonate's gestational age } & \multirow{2}{*}{ Total $(\%)$} \\
\hline & $<37$ weeks $(\%)$ & $>=37$ weeks $(\%)$ & \\
\hline Mother with dengue and neonate born within the MFVP & $6(14.6)$ & $35(85.4)$ & $41(100.0)$ \\
\hline Mother with dengue and neonate Born outside MFVP & $14(4.7)$ & $281(95.3)$ & $295(100.0)$ \\
\hline Mother without dengue & $17,079(4.9)$ & $328.520(95.1)$ & $345,599(100.0)$ \\
\hline Total & $17,099(4.9)$ & $328.836(95.1)$ & $345,935(100.0)$ \\
\hline
\end{tabular}

$\chi^{2}=8.221 ;$ d.f. $=2 ; p=0.016$. MFVP: maternal-fetal viremia period; $\chi^{2}:$ chi-square; d.f.: degree of freedom

Page 2 of 3 
Ribeiro CF, Lopes VGS, Brasil P, Silva LE, Ribeiro PHFJ, Ugenti LC, Nogueira RMR. Dengue during pregnancy: association with low birth weight and prematurity. Rev Inst Med Trop Sao Paulo. 2016;58:8.

Table 2

Association between dengue during gestation and low birth weight

\begin{tabular}{|c|c|c|c|}
\hline \multirow{2}{*}{ Mother/Neonate } & \multicolumn{2}{|c|}{ Low birth weight } & \multirow{2}{*}{ Total (\%) } \\
\hline & Yes $(\%)$ & No $(\%)$ & \\
\hline Mother with dengue and neonate born within MFVP & $9(22.0)$ & $32(78.0)$ & $41(100.0)$ \\
\hline Mother with dengue and neonate born outside the MFVP & $13(4.4)$ & $282(95.6)$ & $295(100.0)$ \\
\hline Mother without dengue & $18,017(5.2)$ & $327,582(94.8)$ & $345,599(100.0)$ \\
\hline Total & $17,099(4.9)$ & $328,836(95.1)$ & $345,935(100.0)$ \\
\hline
\end{tabular}

$\chi^{2}=23.627 ;$ d.f. $=2 ; p<0.0001$. MFVP: maternal-fetal viremia period; $\chi^{2}:$ chi-square; d.f.: degree of freedom

period of 15 days between the date of the first maternal symptoms and the date of birth (maternal-fetal viremia period). In these cases, however, low birth weight could be related to prematurity and not to the intrauterine growth restriction corroborated with literature description, as well as the relation between the presence of DENV infection and increase in prematurity ${ }^{6,7,12}$.

The main limitation of the study is the lack of stillbirth, abortion, and congenital malformation database, which could not be associated to the pregnant women and dengue databases, since the damages of the conceptus found were related only to the third trimester of gestation.

Prematurity and low birth weight were found in cases in which the birth occurred in the MFVP (interval between D1 and labor $\leq 15$ days). This period considers the maternal viremia period (5-6 days), the fetal incubation period (3-5 days), and the fetal viremia period, showing some association between birth in this period and the presence of damages to the conceptus. Similar findings were not found in literature. Some authors, in the last 20 years, have described the harmful effects to the fetal and neonatal health and wellbeing related to the presence of DENV infection in pregnant women, such as the increase in numbers of premature labors, low birth weight related to prematurity, fetal death and conceptus losses ${ }^{5,6,7,8}$. Still in these articles, the results are descriptions of the clinical picture presented by pregnant women and the fetal outcomes, without statistical analysis associating the findings, nor information regarding the considered viremia period, when it is necessary to observe the neonates. The statistically significant association between birth in the MFVP and damage to the conceptus strengthens the necessity to consider the extension of the period of surveillance of maternal viremia, and to include the incubation period in the conceptus and the conceptus viremia period to the outcomes of dengue in pregnancy. Whether DENV infection is associated with poor outcomes in the beginning of pregnancy is not known, however, our study suggests that maternal disease in the final period of gestation can result in damages to the conceptus. Further studies are necessary to conclude if MFVP is the period necessary to either produce an inflammation of the placenta or cause fetal DENV infection that can result in the concept's damage.

\section{CONCLUSION}

The dengue virus infection during gestation may harm the conceptus, and be associated with low birth weight and prematurity.

The risk of damage to the conceptus is associated with the MFVP and not with the maternal viremia period.

\section{ACKNOWLEDGMENTS}

We thank Rômulo Cristovão de Souza, Tizuco Shiraiwa, Gualberto Teixeira dos Santos Junior and Ângela Cascão for their contribution in data collection.

\section{REFERENCES}

1. Halstead SB. Dengue in the Americas and Southeast Asia: do they differ? Rev Panam Salud Publica. 2007;20:407-15.

2. WHO. Dengue and dengue hemorrhagic fever. Geneva: WHO; 2002.

3. Parsonson IM, Della-Porta AJ, Snowdon WA. Developmental disorders of the fetus in some arthropod-borne virus infections. Am J Trop Med Hyg. 1981;30:660-73.

4. Waddy RB. Dengue in early pregnancy. Lancet. 1948;11:950.

5. Alvarenga C, Silami V, Brasil P, Boechat ME, Coelho J, Nogueira RM. Dengue during pregnancy: a study of thirteen cases. Am J Infect Dis. 2009;5:295-300.

6. Basurko C, Carles G, Youssef M, Guindi WEL. Maternal and fetal consequences of dengue fever during pregnancy. Eur J Obstet Gynecol Reprod Biol. 2009; 147:29-32.

7. Carles G, Talarmin A, Peneau C, Bertsch M.Dengue fever and pregnancy. A study of 38 cases in french Guiana. J Gynecol Obstet Biol Reprod (Paris). 2000;29:758-62.

8. Carles G, Peiffer H, Talarmin A. Effects of dengue fever during pregnancy in French Guiana. Clin Infect Dis. 1999;28:637-40.

9. Battaglia FC, Lubchenco LO. A practical classification of newborn infants by weight and gestational age. J Pediatr. 1967;71:159-63.

10. Lubchenco LO, Hansman C, Dressler M, Boyd E. Intrauterine Growth as Estimated from Liveborn Birth-Weight Data at 24 to 42 Weeks of Gestation. Pediatrics. 1963;32:793-800

11. Apgar V. A proposal for a new method of evaluation of the newborn infant. Anesth Analg. 1953;32:260-7.

12. Adam I, Jumaa AM, Elbashir HM, Karsany MS. Maternal and perinatal outcomes of dengue in PortSudan, Eastern Sudan. Virol J. 2010;7:153.

13. Camargo JR KR de, Coeli CM. Reclink: an application for database linkage implementing the probabilistic record linkage method. Cad Saúde Pública. 2000;16:439-47.

Received: 05 February 2015

Accepted: 29 May 2015 